\title{
Cryptographic based Message Transfer using Block chain Technology
}

\author{
A. Krishna Chaitanya ${ }^{1}$, M L Prasanthi ${ }^{2}$, Dr. N Sambasivarao ${ }^{3}$ \\ ${ }^{1}$ Assistant Professor, Department of Information Technology, Institute of Aeronautical Engineering, \\ Hyderabad, India. chaituit2004@gmail.com \\ ${ }^{2}$ Assistant Professor, Department of Information Technology, BVRITH College for Women, Hyderabad, India \\ ${ }^{3}$ Professor, Dept. of Computer Science Engineering, Rishi MS Institute of Engineering and Technology, \\ Hyderabad, India
}

\section{ABSTRACT}

Black chain is a digitized, decentralized, public ledger for constantly on crypto currency transactions. Continually developing 'completed' blocks (the majority later transactions) would recorded and included to it over ordered request it permits advertise members will keep track about advanced cash transactions without focal record keeping. Each hub (a PC associated with the network) gets a duplicate of the piece chain, which will be downloaded naturally. Initially created similarly as those bookkeeping technique to those virtual money spot bit coin, black chains - which utilize what's known as dispersed record innovation organization (DLT) - need aid showing up clinched alongside an assortment for business provisions today. Currently, those innovation is principally utilized with check transactions, inside advanced currencies however it may be could reasonably be expected to digitize code Furthermore embed practically whatever record under the black chain. Completing along these lines makes a permanent record that can't make changed; furthermore, that record's genuineness could make checked by the whole group utilizing those piece chain As opposed to a single unified power. Black chain will be a tamper-evident, imparted advanced record that records transactions in a open alternately private peer-to-peer system. Dispersed will know part hubs in the network, the record lasting press fabric records, for a consecutive chain of cryptographic hash-linked blocks, those history about benefit exchanges that occur between those associates in the system. Every last one of affirmed and approved transaction obstructs are joined and anchored starting with the starting of the chain of the vast majority present block, subsequently the sake piece chain. The black chain In this way goes about Likewise a absolute wellspring of truth, Furthermore parts done a piece chain organize can see just the individuals transactions that are pertinent with them.
Key words: Localization, Ledgers, Cryptograms, Micro payments.

\section{INTRODUCTION}

A block chain is an endlessly developing dispersed database that protects against tamper and review of information. Transactions are added in blocks and must follow the exact order in which they happened (thus the name block chain). Bit coin uses block chain with keep up its state funded record from claiming each single transaction at any point settled on with bit coin. This Merkle tree methodology considers a more terrific hashing system will provide productive Furthermore secure confirmation for a lot for information. This data is that point utilized toward touch coin to implement their transnational checks.

Block chain is not just restricted to the money related system; instead, it will be an incredible result to very nearly whatever stage or item that obliges trust, for example, keyless car passage verification. Additionally, IBM Also Samsung as of late uncovered a evidence of idea that utilize block chain concerning illustration the spine of the internet of things.

The idea behind block chain, over short, will secure also affirm confidence for no those need of a unified framework. Instead, this control might be given on a decentralized network, making it not best that's only the tip of the iceberg secure as well as both that's only the tip of the iceberg proficient Furthermore quicker on scale. A decentralized commercial center might replace ability showcase authority in Ebay, Amazon, and Furthermore Uber. This might imply that trust, rules, identity, reputation, Furthermore installment decisions might make inserted at the client level Furthermore members touch base generally trusted Also recognized for a decentralized manner. There are many applications that are related to Block Chain Technology like Banking, Messaging Funds, Hedge Funds, Voting, Internet Identity and DNS, Internet Advertising, Ride 
Sharing, Crypto Exchanges as shown in the figure below.

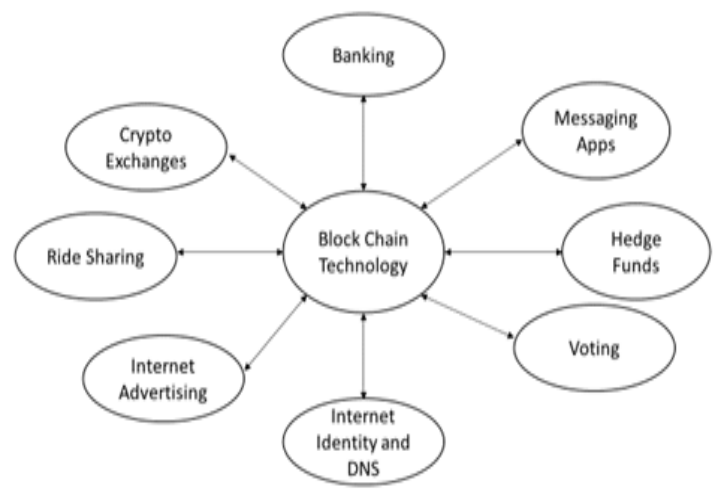

Figure 1: Various domains that support Block Chain Technology.

To today's associated also coordinated circuit world, investment movement takes put in benefits of the business networks that compass national, geographic, Furthermore jurisdictional limits. Business networks regularly meet up In marketplaces the place the participants, for example, such that producers, consumers, suppliers, partners, business sector makers/enablers, And different stakeholders own, control, Also exercise their rights, privileges, and entitlements for Questions of quality known as possessions. Stakes might make unmistakable and physical, for example, such that cars, homes, alternately strawberries, or immaterial holding furthermore virtual, for example, deeds, patents, and stock certificates. Advantage proprietorship Also transfers need aid those transactions that make esteem over a benefits of the business system. Transactions regularly include Different members in buyers, sellers, and go-betweens (such as banks, auditors, or notaries) whose business Agree me also contracts would recorded done ledgers. Benefits of the business normally utilization different ledgers with stay with track for advantage proprietorship and benefit transfer between members in its Different lines for organizations. Ledgers need aid the frameworks of record (SORs) for a business's financial exercises Furthermore hobbies. A disseminated record is a sort for database that is shared, replicated, and synchronized around those parts of a system. The dispersed record records those transactions, for example, the trade of holdings alternately data, around the members in the system. Members in the system oversee Furthermore consent by agreement on the updates of the records in the record. No central, thirdparty mediator, for example, a budgetary organization or clearinghouse, will be included. Each record in the conveyed record need a timestamp Also interesting cryptographic signature, Therefore settling on those record an review equipped history of every one transactions in the organize. Particular case usage for conveyed record innovation is the open sourball hyper record fabric piece chain. Current benefits of the business ledgers being used today are insufficient from various perspectives. They would inefficient, costly, non-transparent, and furthermore liable on duplicity Also abuse. These issues originate from dependence for centralized, trust-based, third-party systems, for example, money related institutions, clearinghouses, and different mediators about existing regulate courses of action. These centralized, trust-based record frameworks prompt bottlenecks and slowdowns from claiming transaction settlements. Absence of transparency, and additionally powerlessness should debasement Also fraud, prompt debate. Hosting to purpose debate Also potentially reverse transactions alternately give protection operator to transactions will be unreason ability. These dangers and uncertainties help missed business chances. Furthermore, out-ofsync duplicates of business ledgers on each organize participant's own frameworks prompt faun benefits of the business choices constructed looking into temporary, inaccurate information. Toward best, the capacity should make a completely educated choice will be deferred same time varying duplicates of the ledgers are determined. As opposed to relying on An third party, for example, a budgetary institution, to intervene transactions, part hubs over An black chain organize utilization An agreement protocol on agrarian for record content, And cryptographic hashes Also advanced marks to guarantee the integument of transactions. Agreement ensures that those imparted ledgers need aid accurate copies, Also lowers those danger for fake transactions, in light of altering might need on happen crosswise over A large number spots during precisely those same chance. Cryptographic hashes, for example, the SHA256 computational algorithm, guarantee that any change should transaction information - Indeed those the vast majority infinitesimal progress - brings about an alternate hash worth being computed, which demonstrates possibly compromised transaction information. Advanced marks guarantee that transactions originated starting with senders (signed for private keys) Furthermore not imposters. Those decentralized peer-to-peer black chain system keeps any solitary member alternately aggregation about members starting with controlling the underlying base alternately undermining the whole framework. Members to organize would constantly on equal, adhering of the same conventions. They might a chance to be individuals, state actors, organizations, or a mix of the sum these sorts about members. At its core, those framework records the ordered request of transactions 
for at hubs agreeing of the legitimacy from claiming transactions utilizing those picked agreement model. That result will be transactions that would irreversible and concurred will toward at parts to organize.

\section{LITERATURE SURVEY}

Block chain is a localized, disseminated record book of all actions which occurs after connecting different parties. It ensures guarantee for all actions as they are unidentified. Each action is checked only after the users agree [1]. Block chain, the base of Bit coin is receiving broad focus recently. Block chain works as a localized record book of all the actions. The applications based on block chain are increasing frequently. The applications like financial services, IOT, reputation systems. There are certain problems in block chain technology like scalability and security has to be affected [5]. Block chain is lately popularized and reformed the digital word by bringing new aspect to the security, efficiency of the systems. Block chain will increase the product innovations and reduces the trade cost. Block chain facilitates the smart contracts, engagements, agreements [2]. Almost, many users confuse block chain with bit coin. But, there is a lot of difference between them Bit coin is just one of the applications that works with block chain technology. Block chain is a form of database from which getting the data is not very easy [3].

Block chain technology is an advanced way in the field of information technology. With Ethereum, block chain will focus on smart contracts, which help in the development of crypto currency [4]. Block chain is a distributed database which keeps a continuously growing tamper proof data structure blocks which holds the individual action as batches. These batches are added in precise and sequential order. Bit coin is a peer-to-peer network which requires no permission and allows every user to connect to the network and send new transaction to send and create blocks [6]. Block chain is a technology that is applied in cryptography to resolve the problems like security and privacy. Block chain is used majorly for privacy and trust [8]. Block chain has witnessed an immense and wide growth in these recent years. There are multiple use cases around its ecosystem. There are numerous attacks on the vulnerable network. Block chain is a peer-to-peer, fault tolerant network that uses puzzles of cryptography to achieve consensus and action management [7]. Block chain has reconstructed the trust definition thus providing the security, integrity and anonymity without need of any third-party. But, there are some disadvantages in the security [9]. Network security and cryptography are the subjects that are ranging widely to show how to protect the digital information [10].

\section{EXISTINGSYSTEM}

An black chain can make considered perfect Likewise a table with three columns, the place each column speaks to An different transaction, the initial section saves those transaction's timestamp, those second section saves those transaction's details, and the third section saves a hash of the present transaction Also its points in addition to the hash of the past transaction. The point when another record may be embedded under a piece chain, the most recent registered hash is broadcasted to each intrigued party. It isn't necessary to each one gathering will stay with a duplicate of the whole transaction history-it's addition that a couple gatherings would. A direct result everybody knows the most recent hash, anybody can check that those information hasn't been modified since it might make incomprehensible without acquiring an alternate And hence invalid hash. That main path to alter for those information same time preserving the hash might a chance to be should find An impact in the data, And that's computationally incomprehensible. It might oblige to such an extent registering control that it's practically uneconomical. An hash might be considered perfect Similarly as a encrypted form of the unique string starting with which it will be difficult on infer the first string. Clinched alongside fact, restricted should figure the hash of a string may be Toward encrypting it And performing a portion scrambling of the yield odds. Mathematically, a hash may be prepared Eventually Tom's perusing An hash function, f, which must need two imperative properties: the span of the enter space and the yield space must make large; it must a chance to be practically incomprehensible with Figure collisions, that is, two inputs $\mathrm{x} 1$ Also $\mathrm{x} 2$ that prepare the same yield $f(x 1) 5 f(x 2)$. An ordinary requisition of hash works will be over secret key storage-when client register looking into a website, client don't have any desire the site to store those watchword $\mathrm{p}$ for its database, generally Any individual with entry of the database Might read it. Those website if store those hash of the password, $\mathrm{f}(\mathrm{p}) 5 \mathrm{y}$. The point when those client login, those enter watchword $\mathrm{p}$ may be hashed once more Also compared with the put away value, $f(p)$ $5 \mathrm{y}$. That likelihood from claiming an inaccurate secret key transforming the same hash quality y Likewise the genuine international ID will be zero for useful purposes. Cases of hash works need aid those secure hash calculations (SHA1, SHA128, SHA512, along these lines on), which would actualized in the standard Python module barbarously. They could make at whatever string Likewise enter and constantly generate a yield string that's a hexa decanoic corrosive representational of the yield number of the work with an altered amount about digits. The population needs a 
constructor, "init", which makes a rundown about pieces Also saves those initially piece in the rundown. Those class likewise need a second method, "record", that, provided for the subtle elements of a new transaction Furthermore a discretionary timestamp (otherwise naturally computed), saves them done another black. This may be carried out Eventually Tom's perusing retrieving the hash of the past piece from self. Blocks, calling those bash function, and appending the triplet (timestamp, details, and new hash) of the rundown about obstructs. Perceive that self. Blocks[i][j] speaks to An cell in the black chain table the place $\mathrm{i}$ is those column amount beginning from 0 , Furthermore $\mathrm{j}$ is those section amount Additionally beginning starting with.

The Work is more on the basic understanding of block chain but when the scenario is considered for crypto currencies like bit coin there's a lot more than this to the bit coin network. The core understanding of block chain adding chain of blocks and validating integrity is more important to be considered in building the Blocks.

\section{PROPOSED METHODOLOGY}

The goal of this paper is to explain and to make clearer how is a block chain structured at the very core. As in figure 2 there are three divisions in implementation: The Message () class, the Block () class and the Chain ().

A message is the basic data container. It is sealed when added to a block and has 2 hashes that identify it: the payload hash and the block hash. Each message is linked to the previous message via hash pointers (the prev_hash attribute). The validate message method will ensure the integrity of each message, but will not check if the hash pointers are correct. This is left to the validate method in the Block () class.

A block can contain $1, \ldots, n$ messages that are linked sequentially one after the other. When a block is added to the chain, it's sealed and validated to ensure that the messages are correctly ordered and the hash pointers match. Once the block is sealed and hashed, it is validated by checking the expected vs the actual.

A chain can contain $1, \ldots, \mathrm{m}$ blocks that are linked sequentially one after another. The chain integrity can be validated at any time calling the validate method, which will call each block's validate method and will raise an Invalid Block chain exception.

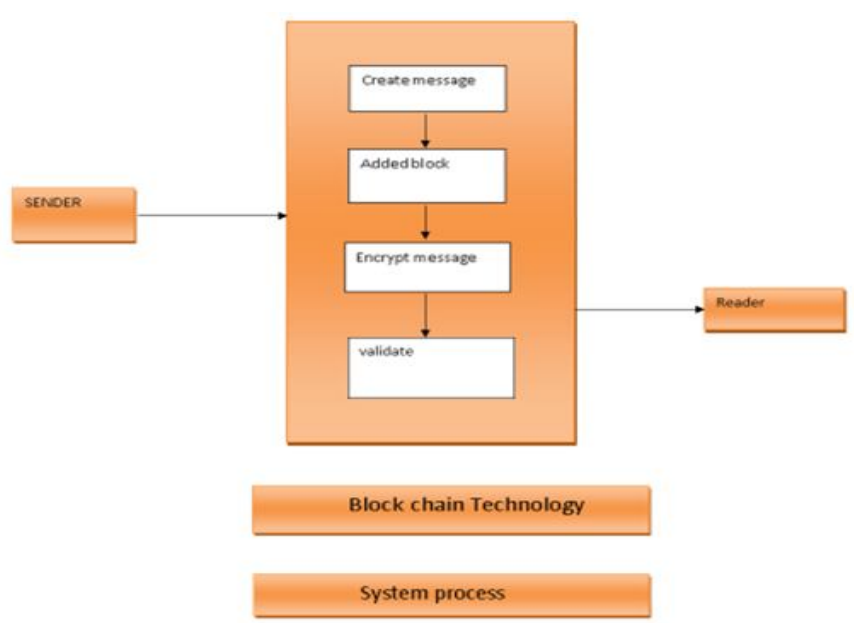

Figure 2: Communication of Sender and Reader with Block chain.

\section{EXPERIMENTAL RESULTS}

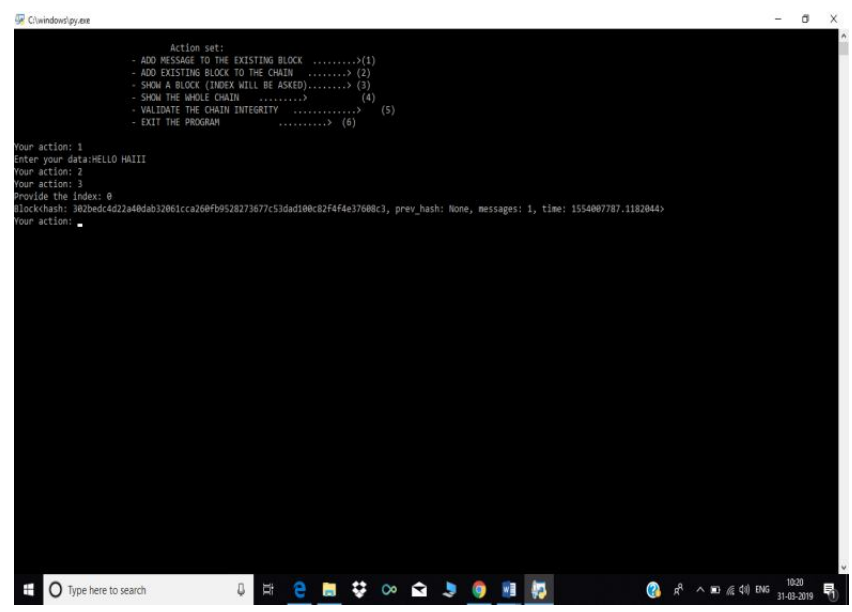

Figure 3: Encryption of Data in Blockchain

A manager () function is provided to interact with the block chain via the Terminal/Console. The basic actions are:

Add Message to Block: Allows adding a message to the current block as in figure 3 .

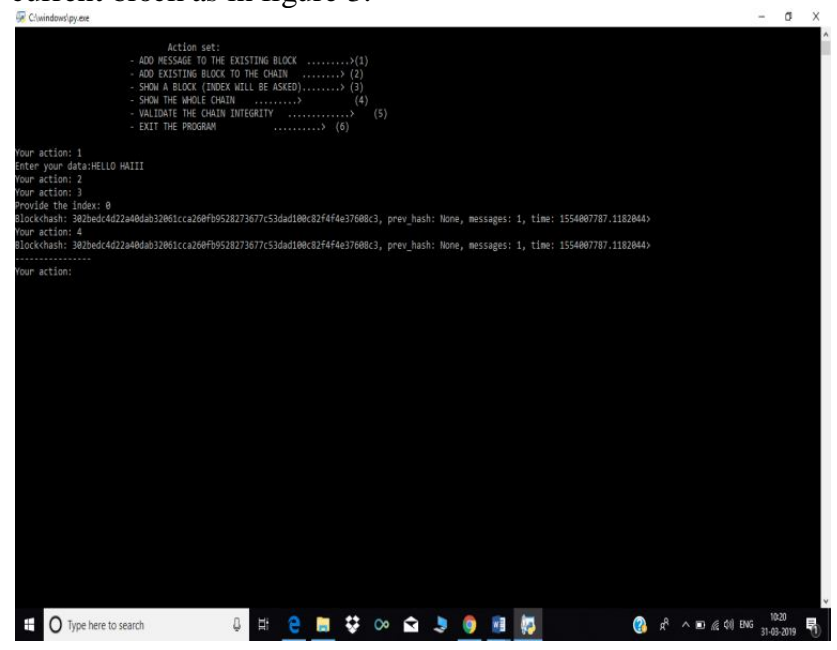

Figure 4: Chain in Blockchain 
Add Block to Chain: Allows adding the current block to the chain if it's not empty.

Show Block: Asks for an index and if exists a block with that index, returns some of the block attributes as in figure 4.

Show Chain: Returns some of the block attributes for each block in the chain.

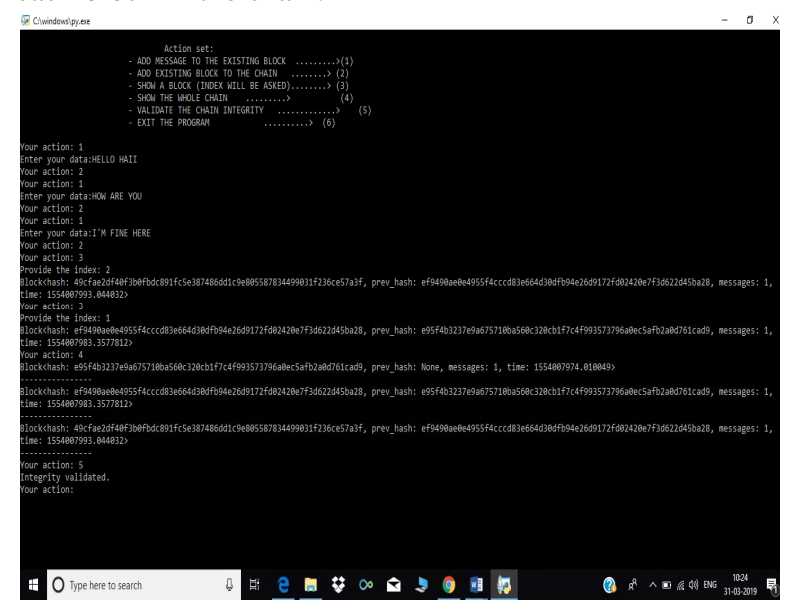

Figure 5: Validating Integrity of Blockchain

Validate Integrity: Returns True if the integrity is validated, terminates the program raising the appropriate exception otherwise as in figure 5.

Exit: Terminates the program and deletes the block chain.

\section{CONCLUSION}

This paper need attempted should show that piece chain technology's a lot of people ideas Also features may make comprehensively extensible should An totally assortment of circumstances. These Characteristics apply not simply of the quick setting from claiming money and installments (Block chain 1.0), alternately should contracts, property, What's more the sum money related business sectors transactions (Block chain 2. 0), However Past should segments Likewise different as government, health, science, literacy, publishing, financial development, art, What's more society (Block chain 3. 0), What's more potentially Indeed going a greater amount comprehensively on empower ordersof-magnitude larger-scale mankind's Advance.

Piece chain innovation Might remain calm integral On a likelihood space for what's to come reality that incorporates both unified Furthermore decentralized models. Similar to any new technology, those square chain will be A thought that at first disrupts, What's more additional time it Might Push those advancement of a bigger biological community that incorporates both those old manner and the new advancement. A percentage authentic cases would that the coming of the radio indeed prompted expanded record sales,
Furthermore e-readers for example, such that the ignite have expanded book bargains. Now, we get news from those New York Times, blogs, Twitter, Also customize ramble encourages indistinguishable. We devour networking starting with both substantial stimulation organizations Also Youtube. Thus, In time, square chain innovation organization Might exist clinched alongside An bigger biological community for both unified Also decentralized models.

\section{REFERENCES}

1. Rishav Chatterjee, Rajdeep Chatterjee,"An Overview of the Emerging Technology: Blockchain",2017 $3^{\text {rd }}$ International Conference on Computational Intelligence and Networks(CINE), October 2017, pp. 126-127. https://doi.org/10.1109/CINE.2017.33

2. TareqAhram,SamanSargolzaei, Jeff Daniels, Ben Amaba, "Blockchain Technolo] gy

Innovations", 2017 IEEE Technology \& Engineering Management Conference (TEMSCON), June 2017. https://doi.org/10.1109/TEMSCON.2017.7998367

3. Pinyaphattasatanattakool, ChianTechapanupreeda“" Blockchain: Challenges and Applications” 2018 International Conference on Information Networking, January 2018, pp.473-475. https://doi.org/10.1109/ICOIN.2018.8343163

4. DejanVujičić, DijanaJagodić, SinišaRanđić "Blockchain Technology, Bitcoin, Ethereum: A Brief Overview" $2018 \quad 17^{\text {th }}$ International Symposium INFOTEH-JAHORINA (INFOTEH) March 2018. https://doi.org/10.1109/INFOTEH.2018.8345547

5. Zibin Zheng, ShaoanXie, Hongning Dai, Xiangping Chen, and HuaiminWang"An Overview of Blockchain Technology: Architecture, Consensus, and Future Trends",6th IEEE International Congress on Big Data, June2017, pp.557-564.

https://doi.org/10.1109/BigDataCongress.2017.85

6. Sachchidanand Singh, Nirmala Singh, "Blockchain: Future of Financial and Cyber Security", 2016 2nd International Conference on Contemporary Computing and Informatics (IC3I), December 2016, pp 463-467.

7. Joanna Moubarak, Eric Filiol, Maroun Chamoun, " On Blockchain Security and Relevant Attacks",2018 IEEE Middle East and North Africa Communications Conference (MENACOMM), April 2018.

https://doi.org/10.1109/MENACOMM.2018.8371010

8. Harry Halpin, Marta Piekarska, "Introduction to Security and Privacy on Blockchain”, 2017 IEEE European Symposium on Security and Privacy Workshops (EuroS\&PW), April 2017, pp 1-3. https://doi.org/10.1109/EuroSPW.2017.43

9. Fangfang Dai, Yue Shi, Nan Meng, Liang 
Wei, Zhiguo Ye, "From Bitcoin to Cybersecurity: A Comparative Study of Blockchain application and Security Issues", 2017 4th International Conference on Systems and Informatics (ICSAI), November 2017, pp.975-979.

10. T. Rajani Devi, "Importance of Cryptography in Network Security”. 2013 International Conference on Communication Systems and Network Technologies, April 2013, pp.462-467.

11. A. Eskicioglu, L. Litwin, "Cryptography." IEEE Potentials Volume: 20,Issue: 1, March 2001, pp.36-38. https://doi.org/10.1109/45.913211

12. Yong Yuan, Fei-Yue Wang "Blockchain and Cryptocurrencies: Model, Techniques and Applications", IEEE Transactions on Systems, Man, and Cybernetics: Systems, Volume:48, Issue:9, July 2018, pp. 1421-1428.
https://doi.org/10.1109/TSMC.2018.2854904

13. Roman Beck, "Beyond Bitcoin: The Rise of Blockchain World", Computer, Volume 51, Issue 2, February 2018, pp. $54-58$. https://doi.org/10.1109/MC.2018.1451660

14. James Carbaugh1, Matthew Fletcher1, Ralucca Gera," Extracting Information Based on Partial or Complete Network Data", International Journal of Advanced Trends in Computer Science and Engineering, Volume 8, No.1.1, 2019.

15. Sungyoung Choi1, Sunmyeng Kim," MultiChannel MAC Protocol based on Dynamic Time Slot Allocation for Underwater Sensor Networks", International Journal of Advanced Trends in Computer Science and Engineering, Volume 8, No.2, March - April 2019.

https://doi.org/10.30534/ijatcse/2019/13822019 